'Departamento de Control de Gestión y Sistemas de Información, Facultad de Economía y Negocios, Universidad de Chile. Santiago, Chile.

${ }^{2}$ Escuela de Auditoria y Control de Gestión, Facultad de Economía y Negocios, Universidad Santo Tomás. Santiago, Chile. ${ }^{a}$ Contador Auditor, Magíster en Control de Gestión.

bingeniero en Información y Control de Gestión, Contador Auditor, Magíster en Finanzas, PhD en Salud Pública.

Fuente de apoyo financiero: Proyecto Fondecyt Iniciación № 11160150. Los autores declaran no tener conflictos de interés.

Recibido el 13 de agosto de 2019, aceptado el 14 de abril de 2020.

Correspondencia a: Alicia Núñez Departamento de Control de Gestión y Sistemas de

Información, Facultad de Economía y Negocios, Universidad de Chile. Diagonal Paraguay 257, of. 2004. Santiago, Chile. anunez@fen.uchile.cl

\section{Indicadores de gestión para evaluar el desempeño de hospitales públicos: Un caso de estudio en Chile y Ecuador}

\author{
JUAN CARLOS ARMIJOS ${ }^{1,2, a}$, ALICIA NÚÑEZ MONDACA ${ }^{1, b}$ \\ Assessing the performance of public \\ hospitals using key indicators: \\ a case study in Chile and Ecuador
}

Background: Continuous improvement, quality of care, and patient satisfaction demand the implementation of coordinated actions from all the healthcare personnel. They also require collaboration, management skills and attention to different dimensions to improve problems due to the lack of resources such as specialists, medical technology and infrastructure. Aim: To design and implement a model of indicators to evaluate the performance of hospitals. Material and Methods: The methodology used in this research included a review of the literature, data collection, conducting interviews, defining objectives and indicators, proposing a model of indicators, validating the set of indicators, implementing the indicators in a hospital, and analyzing the results. Results: The proposed model of 95 indicators was implemented in a hospital in Ecuador. The results indicate that 37 indicators meet the standard, 19 need to be reviewed, 10 show non-compliance and need serious improvements, and the remaining 29 were not informed by the hospital under study. Conclusions: The defined indicators are aimed to improve the performance of a hospital, are easily interpreted, can be measured without spending large amounts of money, and do not need excessive efforts to collect data, mainly if they are supported by information systems.

(Rev Med Chile 2020; 148: 626-643)

Key words: Efficiency; Healthcare; Hospitals, Public; Management Service Organizations; Quality Indicators.
U no de los grandes problemas en salud pública es la percepción de mala gestión en todos sus niveles, lo que genera desconfianza e insatisfacción por parte de quienes hacen uso de los servicios de salud. Por ello, los hospitales deben hacer múltiples esfuerzos para garantizar el cumplimiento de aspectos esenciales para su correcto funcionamiento, asegurando la efectividad, eficiencia y eficacia en el desarrollo de sus actividades. Al respecto, desde hace varios años se plantea la idea de cambiar la metodología utilizada para evaluar con mayor eficacia los centros hospitalarios, como consecuencia de la creciente complejidad en la que se encuentran inmersos.

La complejidad de los hospitales públicos se encuentra asociada con los servicios que presta, que dependen de múltiples procesos. Para llevarlos a cabo, las instituciones deben apoyarse de recursos humanos calificados, recursos económicos acorde a las necesidades, modernos sistemas de información, entre otros ${ }^{1}$. Sin embargo, también se deben considerar aspectos como: 1) la recopilación eficiente de la información; 2) la elaboración de informes para la toma de decisiones y 3 ) la inducción a los empleados para ejecutar acciones alineadas con los objetivos de la organización ${ }^{2}$. 
Para ello, es indispensable realizar una adecuada gestión hospitalaria monitoreando por medio de indicadores. Estos indicadores deberían ser universales, útiles, sencillos de medir e interpretar, para aprovechar de mejor forma su aplicación. Esto permite además comparar rendimientos con diferentes hospitales públicos dentro de un país, o entre diferentes países de una misma región ${ }^{3}$.

A nivel mundial, el uso de indicadores de gestión es cada vez más frecuente. En España, la Sociedad Española de Calidad Asistencial, desde hace varios años, ha desarrollado proyectos encaminados a la elaboración de un "conjunto de indicadores que permitan monitorear e impulsar la mejora continua de la calidad en los hospitales del Sistema Nacional de Salud"4. En Latinoamérica, Brasil (específicamente en Sao Paulo), se busca evaluar el desempeño del personal de enfermería de los hospitales públicos, por medio de indicadores de gestión ${ }^{5}$. En Chile, la Superintendencia de Salud, mediante su página web, provee de una serie de manuales, guías, e informes respecto del uso de indicadores hospitalarios. Entre ellos, indicadores del National Quality Measures Clearinghouse, de la OECD Health Care Quality Indicators, y de la Guía Práctica para el Proceso de Acreditación de Prestadores Institucionales de Salud. Este último, fundamenta su propósito en el cumplimiento de estándares mínimos fijados por el Ministerio de Salud, cuya evaluación es realizada por pares externos, y que permite, la acreditación y certificación hospitalaria ${ }^{6}$. Por su parte, en Ecuador, existen áreas específicas en las que se ha enfocado la aplicación de indicadores hospitalarios. El Manual de Estándares de Indicadores e Instrumentos para medir la Calidad de la Atención Materno-Neonatal, es uno de ellos ${ }^{7}$. Otros datos sobre indicadores se muestran mediante documentos metodológicos presentados por el Instituto Nacional de Estadísticas y Censos, por medio de los cuales se reporta información estadística sobre el registro de camas y egresos hospitalarios $^{8}$. Sin embargo, tanto en Chile, como en Ecuador, países foco de este estudio, aún los ministerios de salud o instituciones competentes no han implementado sistemas para evaluar el desempeño integral de hospitales mediante el uso de indicadores de gestión de los procesos que realizan. Esto queda en evidencia en estudios, que concluyen que aún existen desafíos respecto del uso de indicadores de gestión hospitalaria, entre ellos: a) deficiencias en su cumplimiento respecto de estándares internacionales; b) falta de conocimiento, y por ende implementación de sistemas que sirvan de herramienta para la toma oportuna de decisiones; c) falta de inversión en salud, entre otros ${ }^{9}$.

En conclusión, "los indicadores tienen un papel clave al convertir los datos en información relevante para los responsables de tomar decisiones en el campo de la salud pública"3. Por lo tanto, este artículo propone un modelo de gestión para evaluar el desempeño de hospitales públicos, a través de un conjunto de indicadores de procesos. Este grupo de indicadores fue definido estudiando las realidades de países a nivel mundial, especialmente de Chile y Ecuador, con el fin de guiar a los hospitales hacia una toma de decisiones oportuna y contribuir a una mejora continua de sus procesos. Adicionalmente, el artículo presenta la aplicación de este sistema de indicadores en un hospital público de Ecuador.

\section{Material y Método}

La metodología se desarrolla en dos etapas (Figura 1). La primera etapa de levantamiento de información se realizó a través de visitas de campo, entrevistas, indagación, búsqueda y propuesta de indicadores de gestión relacionados con procesos críticos o claves. La segunda etapa corresponde a la aplicación del modelo en un hospital del Ecuador.

\section{Etapa 1: Levantamiento de Información y mode- lo de indicadores}

La etapa 1 comienza con la revisión bibliográfica de indicadores de procesos (no incluye indicadores clínicos). Esta revisión tuvo como soporte el análisis de libros, manuales, guías, procedimientos, publicaciones e instrumentos legales enfocados en la evaluación del desempeño, análisis de los sistemas de salud de Chile y Ecuador, indicadores de gestión relacionados con procesos críticos para la gestión de hospitales públicos y recopilación de indicadores publicados en sitios web.

La lista inicial incluyó 694 indicadores que posteriormente fue depurada de acuerdo a los siguientes criterios: 1) Eliminación de indicadores duplicados en su naturaleza (428 indicadores restantes);2) Eliminación de indicadores de actividades específicas de una unidad/servicio hospitalario 


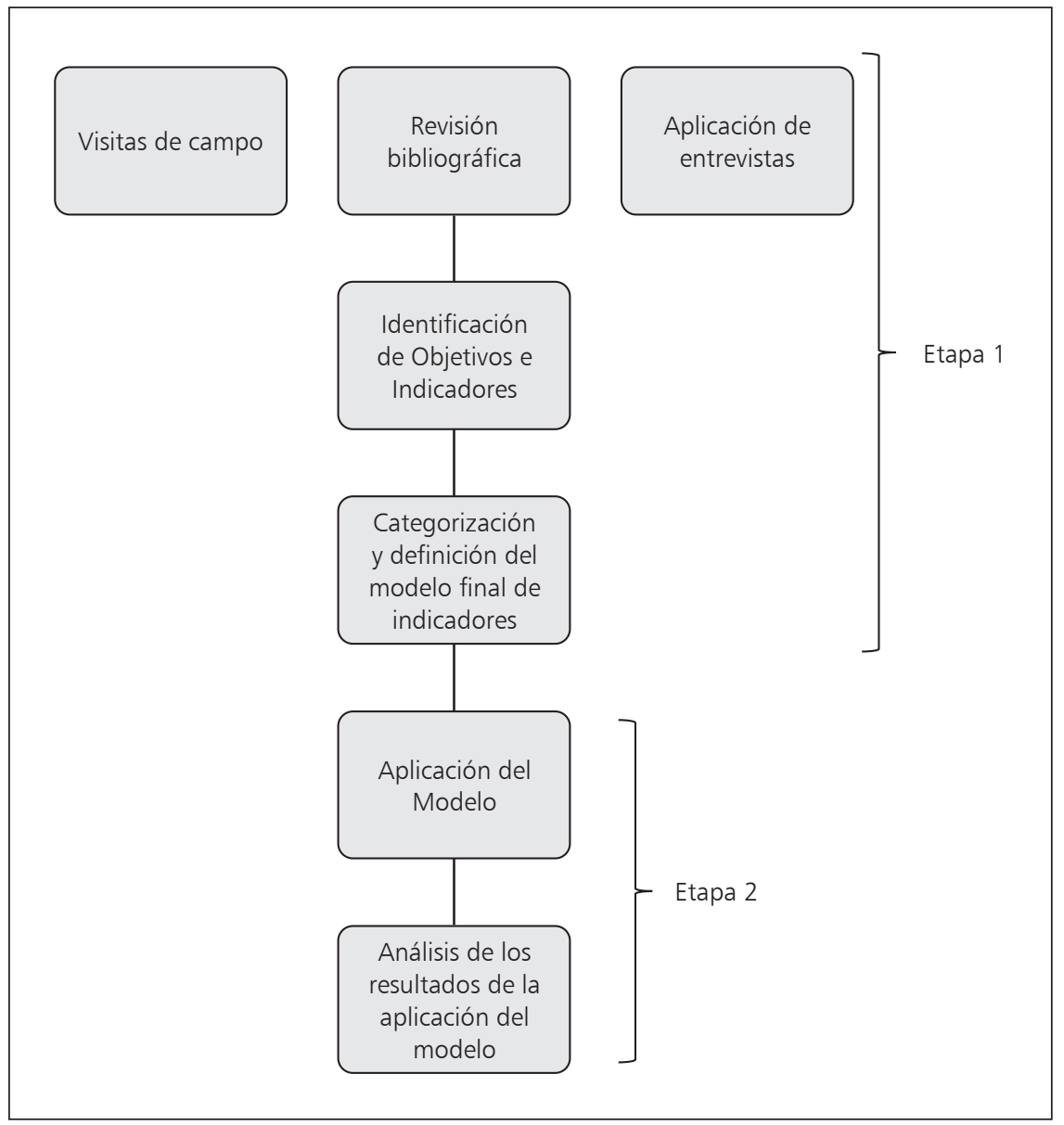

Figura 1. Metodología de trabajo empleada.

(gastroenterología, odontología, UCI, etc.), 250 indicadores restantes; 3 ) Eliminación de indicadores de desempeño de hospitales especializados en áreas de la medicina (cáncer, psiquiatría, pediatría, entre otras), 160 indicadores restantes; 4) Criterio experto, mediante la colaboración de 15 profesionales, entre ellos: directora zonal de salud (Ecuador), directores médicos (Chile y Ecuador), jefes departamentales, médicos, enfermeras, y personal administrativo. El criterio experto redujo el listado a 73 indicadores. 5) Agregación de 11 indicadores relevantes para la gestión hospitalaria de acuerdo a criterio experto. De esta forma, el modelo final incluye un total de 84 indicadores de gestión.

Los indicadores seleccionados se agruparon en base a categorías y subcategorías $(10,11,3)$ : Indicadores de Tiempo (tiempos de espera/ proceso), Calidad (errores/cumplimiento de es- tándares/satisfacción), Eficiencia (productividad (rendimiento)/cumplimiento de estándares), Capacidad (oferta/demanda) y Económicos (costos/ financieros) (Tabla 1).

Es importante mencionar que cada uno de los indicadores tiene asociado un objetivo, una métrica, un estándar, una frecuencia, y un área o departamento a cargo. El objetivo determina el fin al que se desea llegar. La métrica establece la fórmula de medición. El estándar determina el grado de cumplimiento esperado para cada uno de los indicadores. La frecuencia señala la periodicidad con la que se realiza la medición de cada indicador. Por último, el área, corresponde a las unidades o departamentos presentes en los hospitales (Unidades Médicas, Neonatología, Emergencias, Cirugía, Calidad, Talento Humano, Finanzas, etc.). Los responsables a cargo de estas 
Tabla 1. Selección y agrupación de los indicadores

\begin{tabular}{|lll|}
\hline Nombre & Tipo & n \\
\hline Indicadores de & Tiempo de espera & 11 \\
tiempo & Tiempo de proceso & 09 \\
Indicadores de & Errores & 18 \\
calidad & Cumplimiento de estándares & 03 \\
& Satisfacción & 10 \\
Indicadores de & Productividad - Rendimiento & 10 \\
eficiencia & Cumplimiento de estándares & 03 \\
Indicadores de & Oferta & 08 \\
capacidad & Demanda & 07 \\
\hline Indicadores & Costos & 03 \\
económicos & Financieros & 02 \\
\hline Total & & 84 \\
\hline
\end{tabular}

Fuente: Elaboración propia.

unidades, serán los encargados de la recopilación y el uso correcto de la información, así como de monitorear y dar cumplimiento a cada uno de los objetivos establecidos.

\section{Etapa 2: Aplicación de Indicadores}

El modelo de indicadores fue aplicado a un hospital público de Ecuador, perteneciente al Sistema de Servicios de Salud del Ministerio de Salud Pública. Este hospital presta atención de salud integral de tipo ambulatorio y de hospitalización para la población de la Región Sur del país. Es, además, un centro de formación profesional de personal médico y de enfermería, así como de investigación bio-social. Su complejidad es de nivel 2, con una dotación de 243 camas destinadas a la atención de una población urbana de 110.633 (76,6\%) habitantes, y de $33.860(23,4 \%)$ para el área rural.

La información para la medición de los indicadores de gestión del hospital fue recolectada durante los meses de febrero y marzo del año 2017. Se determinaron umbrales para cada indicador utilizando la técnica de semaforización tradicional. La semaforización ayuda a determinar el grado de cumplimiento de cada indicador, siendo así, que, para el color verde (cumplimiento del indicador), se establece un índice $(>=90)$, para el amarillo (precaución o revisión del proceso) $(>=70 \mathrm{y}$
$<90$ ), para el rojo (incumplimiento) $(<70)$, incluyendo un color (gris), para identificar aquellos indicadores sin registro de información. El criterio utilizado para la medición de los indicadores de tiempo se estableció de la siguiente forma: para el color verde $(<=\mathrm{t})$, para el amarillo $(>\mathrm{t} y<\mathrm{t}$ $+5 \mathrm{~min}$ ), y para el color rojo ( $>\mathrm{t}+5 \mathrm{~min})$. Los estándares asociados a cada uno de los indicadores fueron establecidos según los criterios obtenidos de los manuales, guías, o informes utilizados para este estudio, recopilados durante la revisión bibliográfica. Los estándares deberían ser ajustados con el estándar establecido por cada institución.

Es importante señalar que, la información para los indicadores de tiempo fue recogida en su totalidad por medio de un cronómetro. Los tiempos fueron tomados de forma aleatoria (varias veces en distintos días de la semana, y en distintas jornadas de atención al público). Por ejemplo, lunes por la mañana, miércoles por la tarde, viernes por la noche, y así sucesivamente.

Para el levantamiento de información de los indicadores de satisfacción fue necesaria la elaboración y aplicación de encuestas, tanto del personal como de los pacientes. Para la elaboración de la encuesta se tomaron en cuenta diversas variables establecidas en la teoría de dos factores de Herzberg, por medio de la cual se presentan, los factores higiénicos o extrínsecos relacionados con la insatisfacción, los cuales engloban todas las condiciones del ambiente en el cual se mueve la persona, y los factores motivacionales o intrínsecos, vinculados con aspectos más profundos respecto del puesto específico que posee el individuo.

En cuanto a los indicadores de eficiencia, calidad, y capacidad, su información fue obtenida mediante la aplicación de entrevistas realizadas al personal de los departamentos de estadística, planificación, y talento humano, además de la revisión de documentos, específicamente, informes y reportes presentados por las unidades del hospital. En relación con el grupo de indicadores económicos, la información fue proporcionada por el departamento de planificación y financiero de la institución.

\section{Resultados}

El listado de indicadores se detalla en la Tabla 2, junto con los resultados obtenidos de la aplicación. 


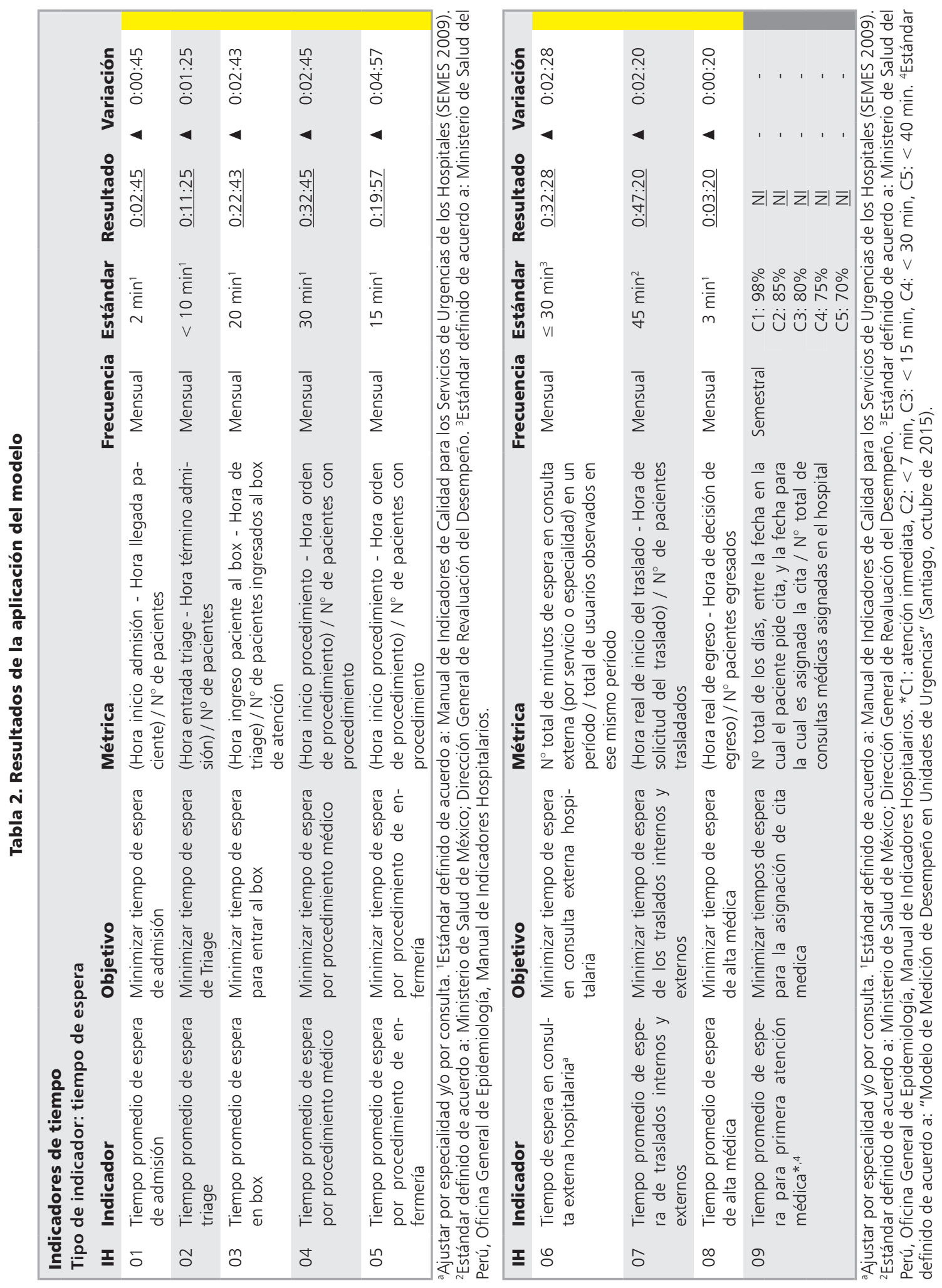




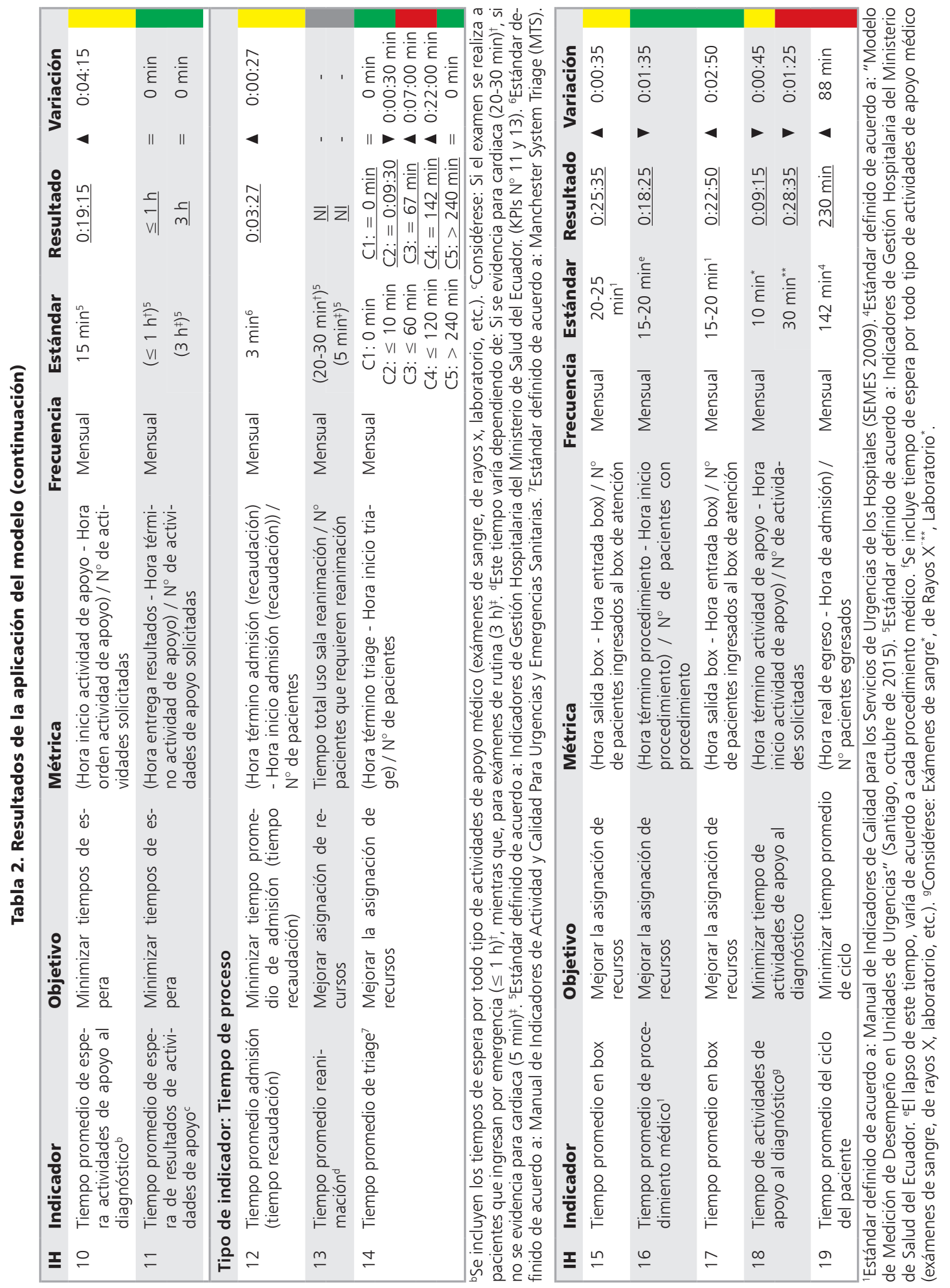



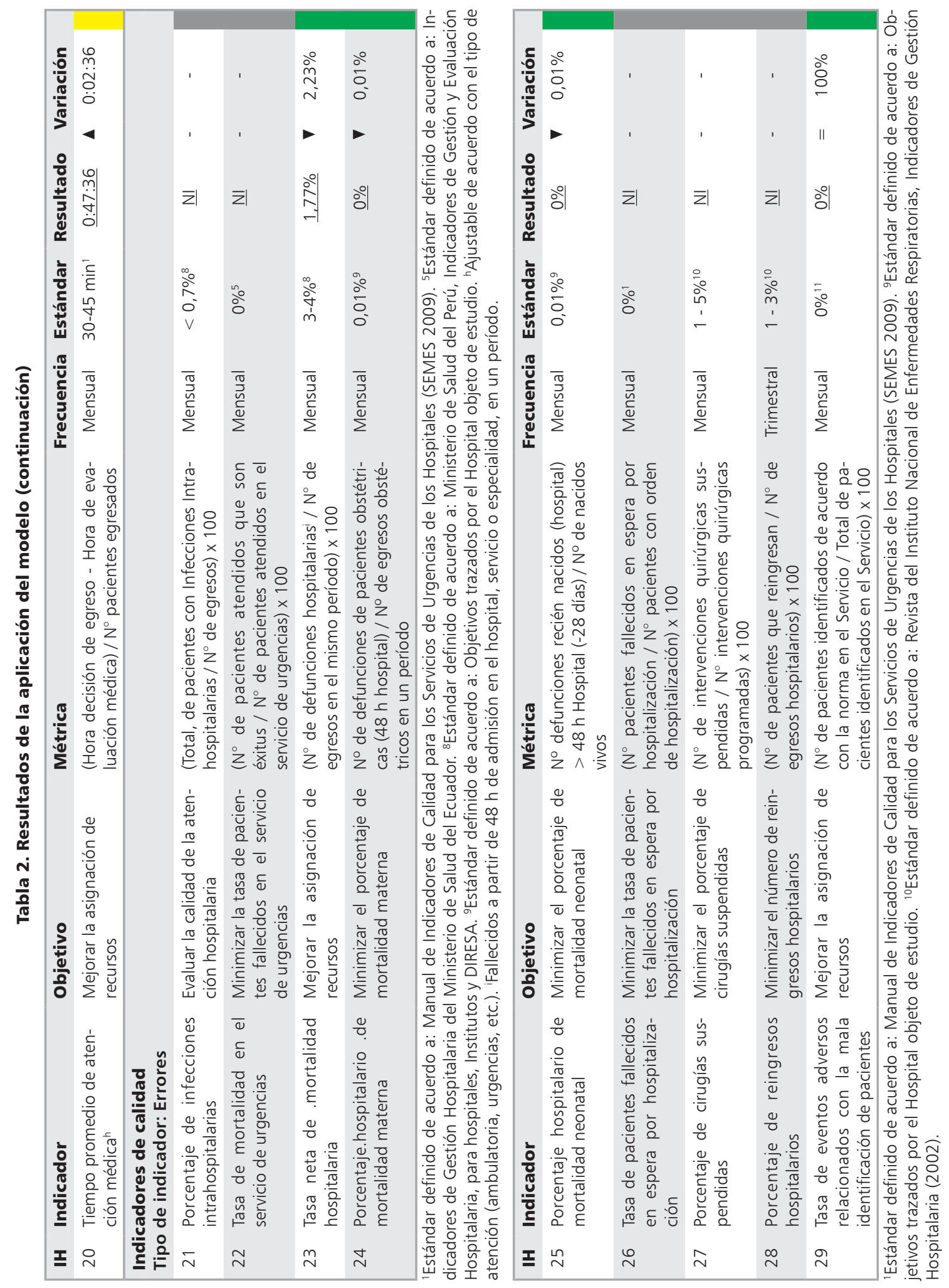

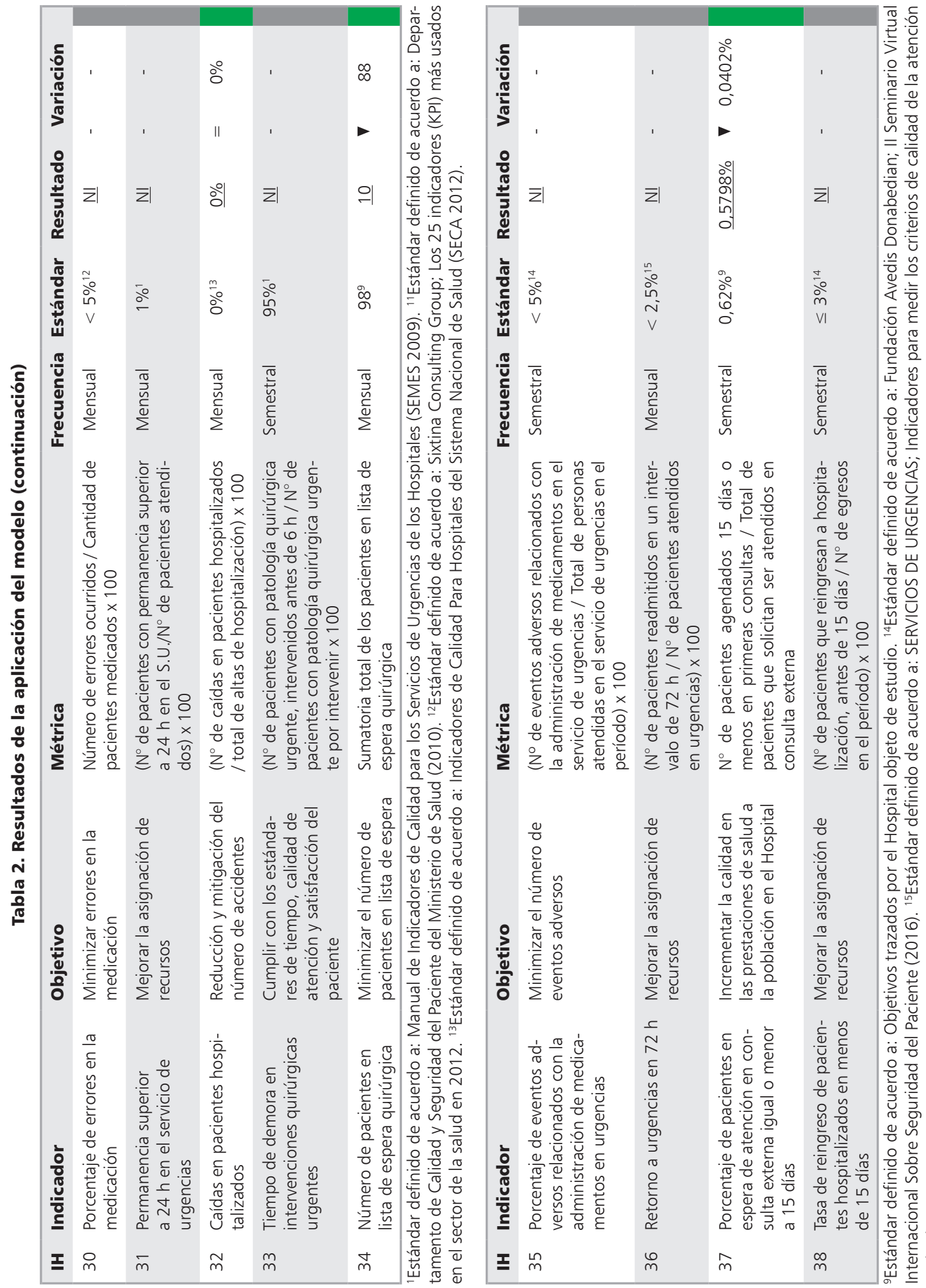

$\circ \frac{0}{0} \frac{1}{4}$

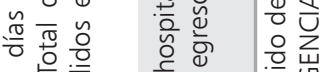

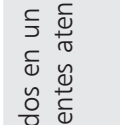
$\stackrel{-1}{-}$ ก

을

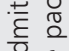

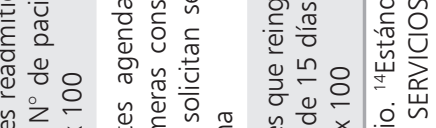

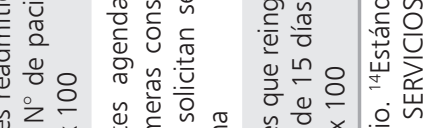

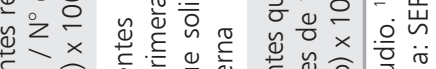

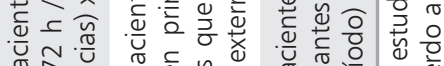

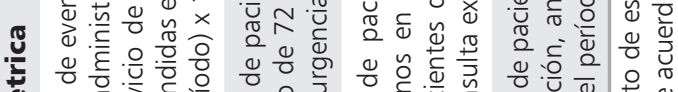

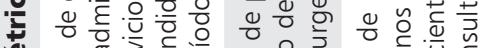

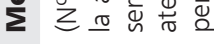
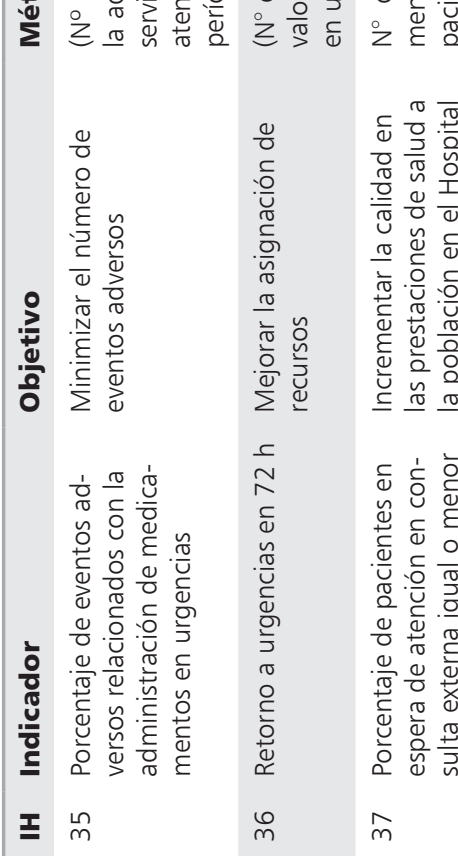

壳
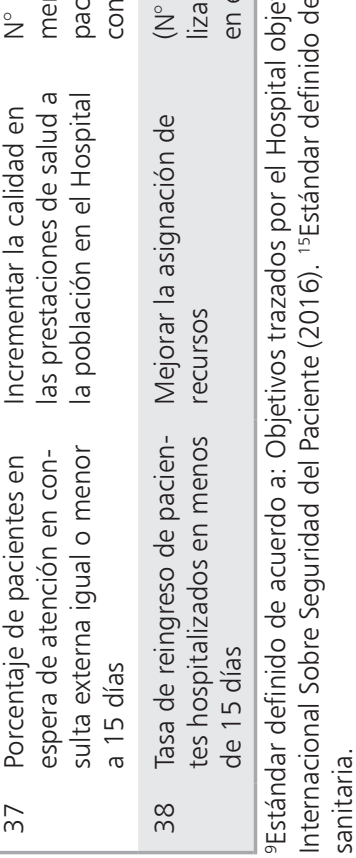


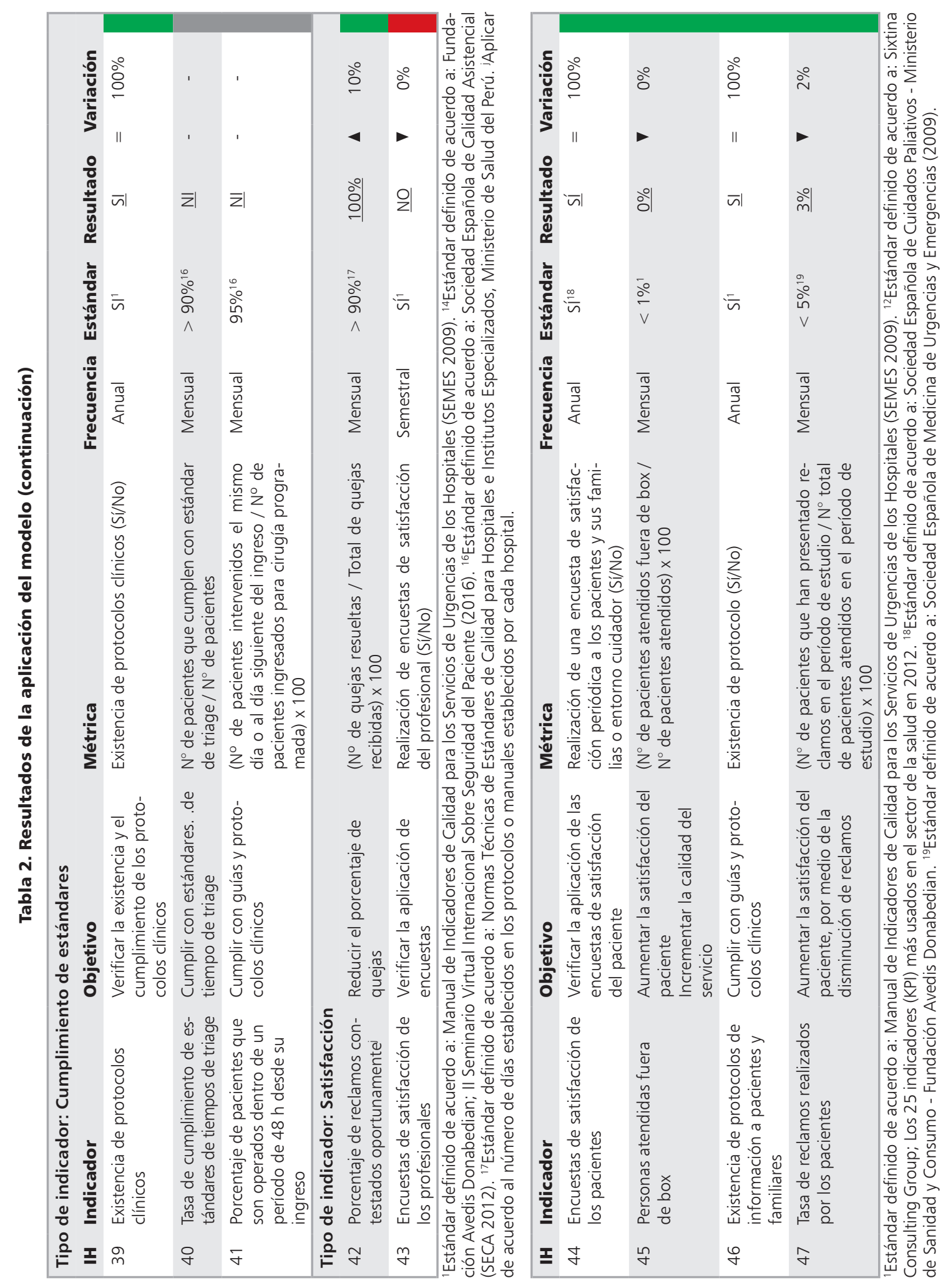




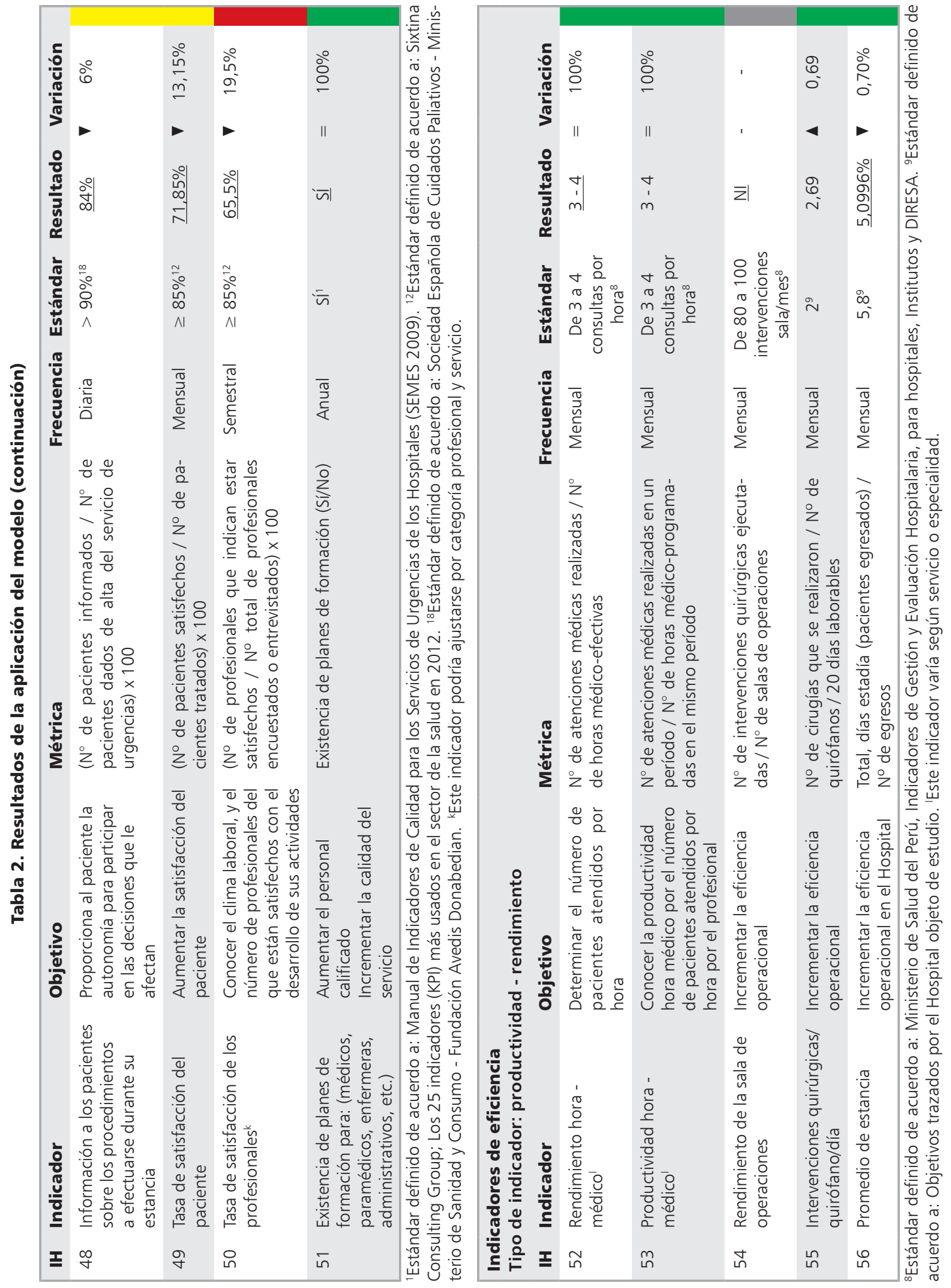




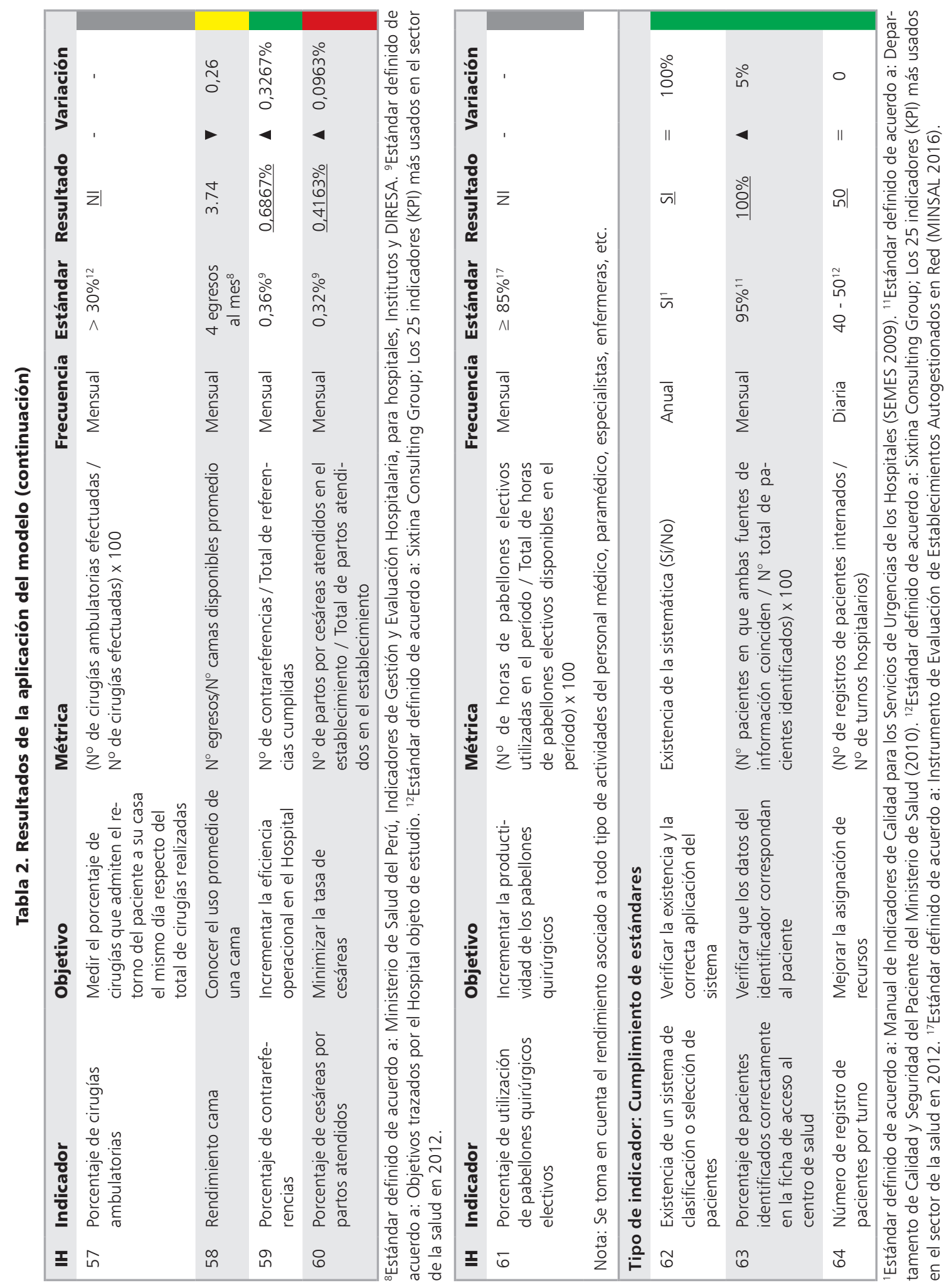




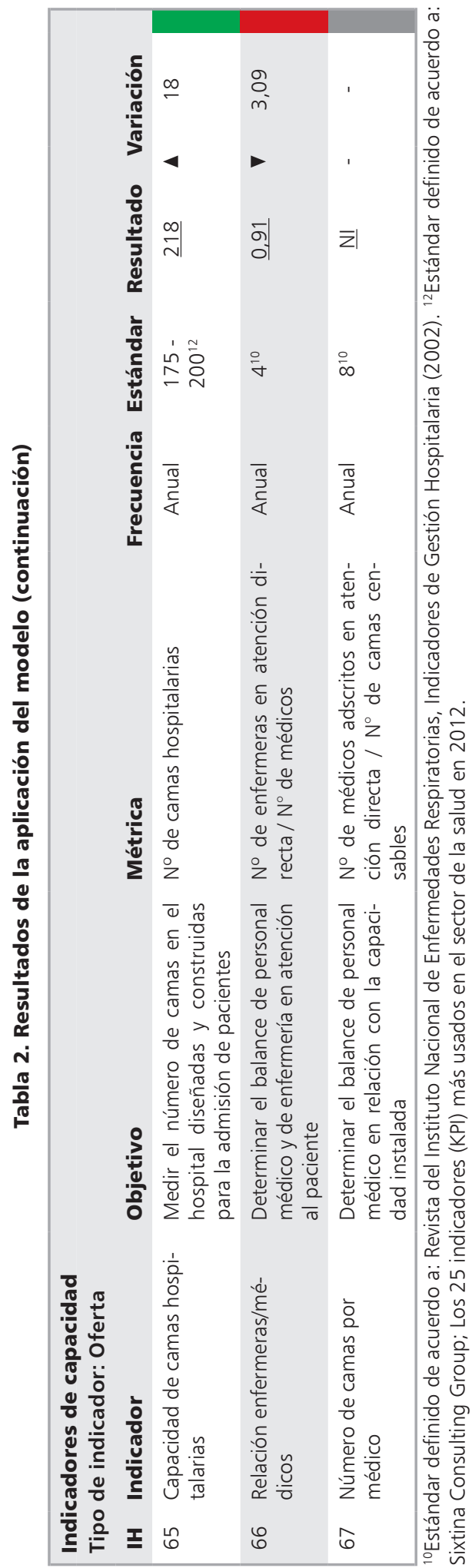

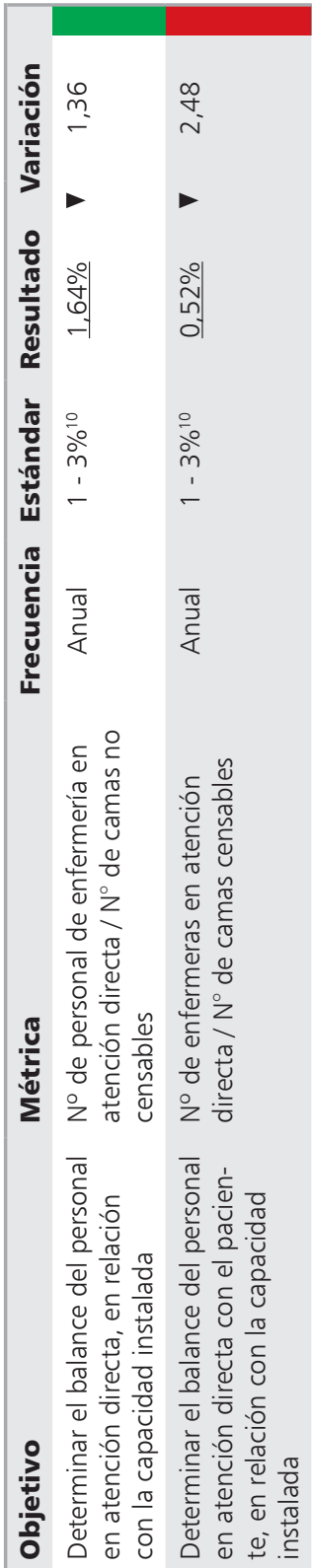

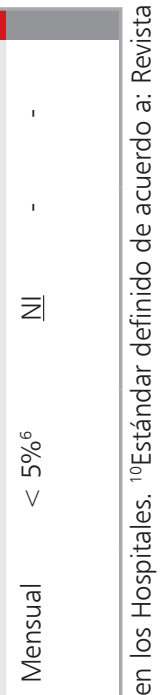

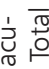

드은

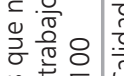

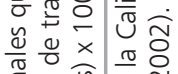

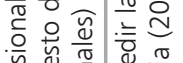

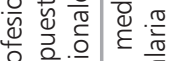

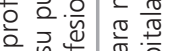

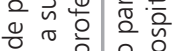
之웡 잉 응 웅

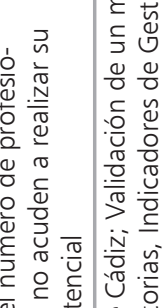

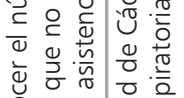
은 บํ.

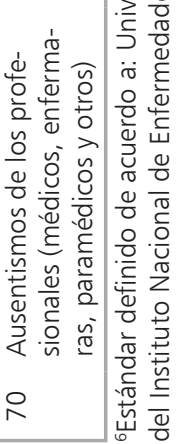

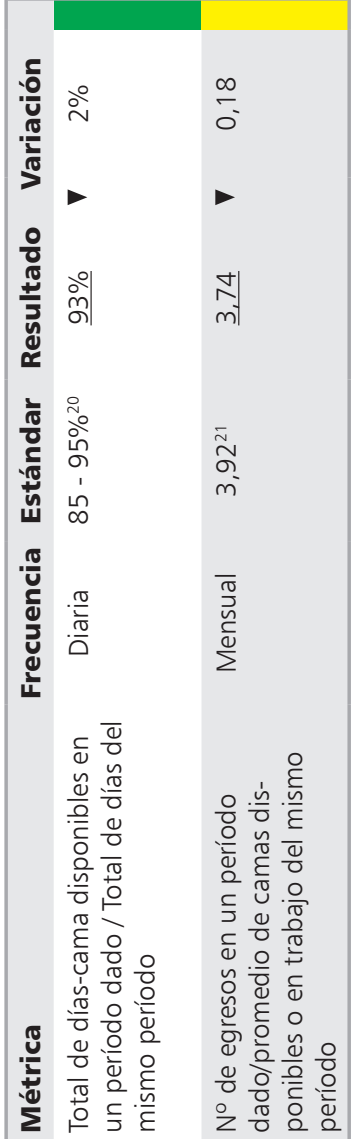

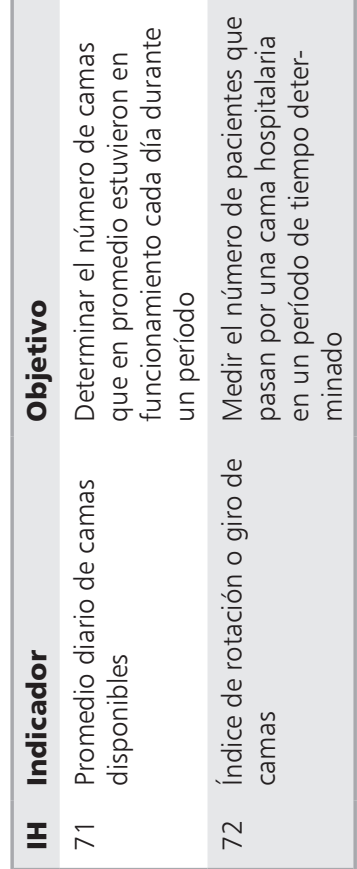



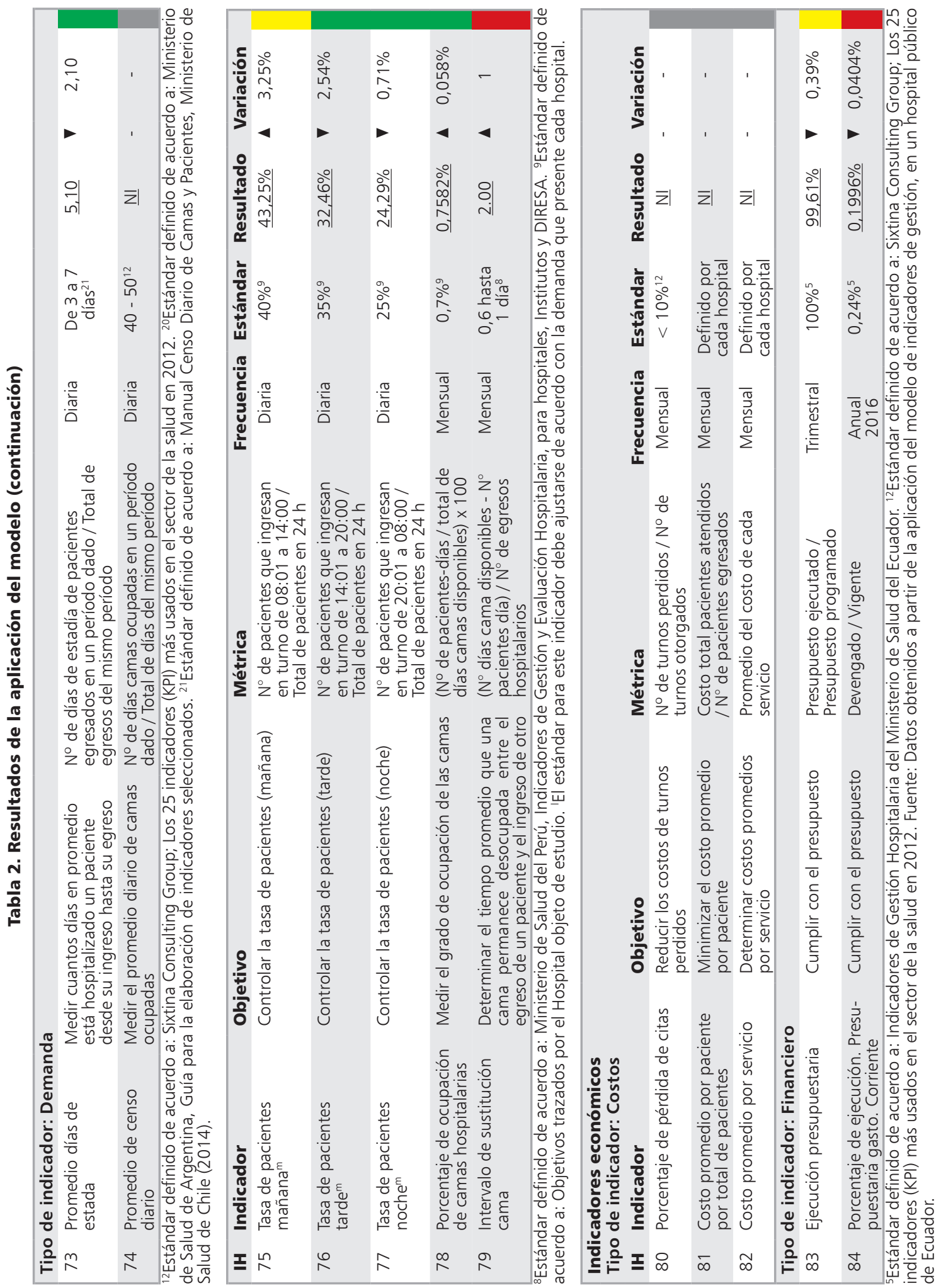
Existen indicadores que por su naturaleza de aplicación se dividen entre sí, como es el caso del tiempo promedio de espera para primera cita médica, del tiempo promedio de triage $(\mathrm{Cl}$ a C5), el tiempo promedio de espera de resultados de actividades de apoyo, tiempo de actividades de apoyo al diagnóstico, y por último el tiempo promedio de reanimación, dando como resultado un total de 95 indicadores.

La Tabla 3 presenta los indicadores de gestión hospitalaria agrupados por Área o Unidad Responsable.

Tabla 3. Agrupación de indicadores por área o unidad responsable

\begin{tabular}{|c|c|}
\hline Área responsable & Indicadores de gestión hospitalaria \\
\hline Unidades médicas & $\begin{array}{l}\text { Tiempo promedio de espera de admisión } \\
\text { Tiempo de espera para triage } \\
\text { Tiempo promedio de espera box } \\
\text { Tiempo promedio de espera por procedimiento médico } \\
\text { Tiempo promedio de espera por procedimiento de enfermería } \\
\text { Tiempo de espera en consulta externa hospitalaria } \\
\text { Tiempo promedio de espera de traslados internos y externos } \\
\text { Tiempo promedio de espera de alta médica } \\
\text { Tiempo promedio de espera para primera cita médica: C1: atención inmediata, C2: < } 7 \text { min, } \\
\text { C3: < } 15 \text { min, C4: }<30 \text { min, C } 40 \text { min } \\
\text { Tiempo promedio de espera de actividades de apoyo al diagnóstico (exámenes de sangre, de rayos } \\
\text { x, laboratorio, etc.) } \\
\text { Tiempo promedio de espera de resultados de actividades de apoyo } \\
\text { Considérese: a Exámenes para pacientes en sala de emergencias } \leq 1 \text { h y b) exámenes de rutina = } 3 \text { h } \\
\text { Tiempo promedio admisión (tiempo recaudación) } \\
\text { Tiempo promedio de reanimación } \\
\text { Tiempo promedio de triage } \\
\text { Tiempo promedio en box } \\
\text { Tiempo promedio de procedimiento médico } \\
\text { Tiempo promedio de procedimiento de enfermería } \\
\text { Tiempo de actividades de apoyo al diagnóstico (exámenes de sangre, de rayos X, laboratorio } \\
\text { Tiempo promedio del ciclo del paciente) } \\
\text { Tiempo promedio de atención médica } \\
\text { Porcentaje de infecciones intrahospitalarias } \\
\text { Tasa neta de mortalidad hospitalaria } \\
\text { Porcentaje hospitalaria de mortalidad materna } \\
\text { Tasa de pacientes fallecidos en espera por hospitalización } \\
\text { Porcentaje de reingresos hospitalarios } \\
\text { Porcentaje de errores en la medicación } \\
\text { Porcentas en pacientes hospitalizados } \\
\text { Tasa de reingreso de pacientes hospitalizados en menos de } 15 \text { días } \\
\text { Tasa de cumplimiento de estándares de tiempos de triage }\end{array}$ \\
\hline Unidades médicas & $\begin{array}{l}\text { Porcentaje de reclamos contestados oportunamente } \\
\text { Encuestas de satisfacción de los pacientes } \\
\text { Personas atendidas fuera de box } \\
\text { Existencia de protocolos de información a pacientes y familiares } \\
\text { Porcentaje de pacientes informados sobre los procedimientos a efectuarse durante su estancia } \\
\text { Rendimiento hora - médico } \\
\text { Productividad hora - médico }\end{array}$ \\
\hline
\end{tabular}




\begin{tabular}{|c|c|}
\hline & $\begin{array}{l}\text { Promedio de estancia } \\
\text { Rendimiento cama } \\
\text { Porcentaje de contrareferencias } \\
\text { Existencia de un sistema de clasificación o selección de pacientes } \\
\text { Porcentaje de pacientes identificados correctamente en la ficha de acceso al centro de salud } \\
N^{\circ} \text { de registros de pacientes por turno } \\
\text { Índice de rotación o giro de camas } \\
\text { Porcentaje de pérdidas de citas } \\
\text { Porcentaje hospitalario de mortalidad neonatal }\end{array}$ \\
\hline Neonatología & Porcentaje hospitalario de mortalidad neonatal \\
\hline Emergencias & $\begin{array}{l}\text { Tasa de mortalidad en el Servicio de Urgencias } \\
\text { Permanencia superior a } 24 \mathrm{~h} \text { en el Servicio de Urgencias } \\
\text { Porcentaje de eventos adversos relacionados con la administración de medicamentos en urgencias } \\
\text { Retorno a urgencias en } 72 \mathrm{~h}\end{array}$ \\
\hline Cirugía & $\begin{array}{l}\text { Porcentaje de cirugías suspendidas } \\
\text { Tiempo de demora en intervenciones quirúrgicas urgentes } \\
\text { Número de pacientes en lista de espera quirúrgica } \\
\text { Porcentaje de pacientes que son operados dentro de un periodo de } 48 \text { h desde su ingreso } \\
\text { Rendimiento de sala de operaciones } \\
\text { Intervenciones quirúrgicas/quirófano/día } \\
\text { Porcentaje de cirugías ambulatorias } \\
\text { Porcentaje de cesáreas por partos atendidos } \\
\text { Porcentaje de utilización de pabellones quirúrgicos electivos }\end{array}$ \\
\hline Calidad & $\begin{array}{l}\text { Existencia de protocolos clínicos } \\
\text { Tasa de reclamos realizados por los pacientes } \\
\text { Tasa de satisfacción del paciente } \\
\text { Capacidad de camas hospitalarias }\end{array}$ \\
\hline Calidad & $\begin{array}{l}\text { Número de camas por médico } \\
\text { Relación enfermeras/camas no censables } \\
\text { Relación enfermeras/camas censables } \\
\text { Promedio diario de camas disponibles } \\
\text { Promedio días de estadía } \\
\text { Promedio de censo diario } \\
\text { Tasa de pacientes mañana } \\
\text { Tasa de pacientes tarde } \\
\text { Tasa de pacientes noche } \\
\text { Porcentaje de ocupación de camas hospitalarias } \\
\text { Intervalo de sustitución cama }\end{array}$ \\
\hline Talento humano & $\begin{array}{l}\text { Encuestas de satisfacción de los profesionales } \\
\text { Tasa de satisfacción de los profesionales (encuesta aplicada por el autor) } \\
\text { Existencia de planes de formación (médicos, paramédicos, enfermeras, administrativos, etc.) } \\
\text { Relación enfermeras/médicos } \\
\text { Relación enfermeras/camas no censables } \\
\text { Relación enfermeras/camas censables } \\
\text { Ausentismos de los profesionales (médicos, enfermeras, paramédicos y otros) }\end{array}$ \\
\hline Finanzas & $\begin{array}{l}\text { Porcentaje de pérdida de citas } \\
\text { Costo promedio por paciente por total de pacientes } \\
\text { Costo promedio por servicio } \\
\text { Ejecución presupuestaria } \\
\text { Porcentaje de ejecución presupuestaria - gasto corriente }\end{array}$ \\
\hline
\end{tabular}

Fuente: Elaborado por los autores. 
Tabla 4. Resultados obtenidos a partir de la aplicación del modelo mediante la técnica de semaforización

\begin{tabular}{|c|c|c|c|c|c|c|c|}
\hline Nombre & Tipo & $\mathbf{n}$ & & & & & $\%$ \\
\hline \multirow[t]{2}{*}{ Indicadores de tiempo } & Tiempo de espera & 16 & $12,5 \%$ & $56,25 \%$ & - & $31,25 \%$ & 100 \\
\hline & Tiempo de proceso & 15 & $40 \%$ & $26,67 \%$ & $20 \%$ & $13,33 \%$ & 100 \\
\hline \multirow[t]{3}{*}{ Indicadores de calidad } & Errores & 18 & $38,89 \%$ & - & - & $61,11 \%$ & 100 \\
\hline & Cumplimiento de estándares & 03 & $33,33 \%$ & - & - & $66,67 \%$ & 100 \\
\hline & Satisfacción & 10 & $60 \%$ & $20 \%$ & $20 \%$ & - & 100 \\
\hline \multirow[t]{2}{*}{ Indicadores de eficiencia } & Productividad - rendimiento & 10 & $50 \%$ & $10 \%$ & $10 \%$ & $30 \%$ & 100 \\
\hline & Cumplimiento de estándares & 03 & $100 \%$ & - & - & - & 100 \\
\hline \multirow[t]{2}{*}{ Indicadores de capacidad } & Oferta & 08 & $37,5 \%$ & $12,5 \%$ & $25 \%$ & $25 \%$ & 100 \\
\hline & Demanda & 07 & $57,14 \%$ & $14,29 \%$ & $14,29 \%$ & $14,29 \%$ & 100 \\
\hline \multirow[t]{2}{*}{ Indicadores económicos } & Costos & 03 & - & - & - & $100 \%$ & 100 \\
\hline & Financieros & 02 & - & $50 \%$ & $50 \%$ & - & 100 \\
\hline Total & & 95 & $39,03 \%$ & $17,25 \%$ & $12,66 \%$ & $31,06 \%$ & 100 \\
\hline
\end{tabular}

Fuente: Datos obtenidos a partir de la aplicación del modelo de indicadores de gestión.

Del $100 \%$ de indicadores presentes en este estudio, 39,03\% corresponden a indicadores de color verde, $18,45 \%$ son indicadores de color amarillo, 11,45\% son indicadores de color rojo, y finalmente $31,06 \%$ de los indicadores tienen un color gris (el hospital no recolecta actualmente ésta información, o no fue informada durante el levantamiento de información) (Tabla 4). Entre los motivos están: falta de información, falta de tiempo, disposición y poca facilidad en la entrega de información por parte del personal, entre otras.

Todos los resultados fueron compartidos y analizados con los responsables del hospital de Ecuador para una posterior definición de estrategias que ayuden a mejorar aquellas áreas más débiles detectadas en este estudio.

\section{Discusión}

Esta propuesta de indicadores es una guía para mejorar la toma de decisiones en hospitales públicos fomentando mejoras en el desempeño y el uso eficiente de los recursos a través de la implementación de indicadores de gestión. La información entregada por este set de indicadores permite enfocar al hospital en medidas correctivas o mejoras en procesos para lograr el cumplimiento de sus objetivos.
Los indicadores propuestos en esta investigación fueron revisados por un grupo de expertos de Chile y Ecuador. Estos indicadores están orientados a la mejora, son fáciles de interpretar, y pueden ser medidos sin grandes recursos, sin embargo, requieren de sistemas de información que apoyen la recolección de datos. Esta última apreciación se realiza ya que durante la aplicación del modelo en el hospital público de Ecuador no fue posible obtener toda la data que permitiese el cálculo de la totalidad de los indicadores propuestos en el modelo. Sumado a ello, es importante mencionar que todos los indicadores tienen un nivel de importancia, relevancia y jerarquía, que depende de la realidad local de cada institución de salud, de manera que respondan a las estrategias que han definido. Sin embargo, aquellos indicadores centrados en el paciente, deben ser aplicados con una mayor precisión y cuidado, como, por ejemplo, aquellos agrupados en indicadores de calidad, y de tiempo de atención de los pacientes.

La recopilación de información a través de estos indicadores da luces respecto de la situación de la institución. En este punto es fundamental recalcar la importancia del compromiso de todos los niveles que conforman la organización, de ellos dependerá: la veracidad de los datos, la confiabilidad y oportunidad de la información y 
el uso adecuado de estos indicadores para tomar acciones específicas de mejora del desempeño organizacional.

Entre los procesos claves a mejorar en el hospital de Ecuador se encuentran aquellos que arrojaron indicadores con resultado rojo, amarillo y gris. Un indicador amarillo, por ejemplo, fue la tasa de satisfacción del paciente, los factores determinantes para que este indicador no cumpliese con su estándar están relacionados con la falta de personal y los largos tiempos de espera para la atención. Todos estos aspectos fueron mencionados por los usuarios, a través de la aplicación de encuestas. Otro punto a mejorar es el relacionado con la tasa de satisfacción profesional. El resultado de las encuestas permitió evidenciar un gran nivel de insatisfacción en torno a la remuneración y beneficios, condiciones de trabajo, relaciones interpersonales, seguridad laboral, premios, incentivos y capacitación, y el relacionado con el crecimiento y desarrollo profesional. Por su parte, en cuanto a los indicadores relacionados con el tiempo promedio de procedimientos de enfermería y atención médica, rendimiento cama, relación enfermeras/ médicos, relación enfermeras/camas censables, porcentaje de ejecución presupuestaria/gasto corriente, entre otros, demuestran posibilidades concretas de mejora, especialmente en torno a la utilización y uso eficiente de los recursos. Es importante dar énfasis también a aquellos indicadores que no fueron informados o que no son aplicados por la institución (31,05\%, del total de indicadores del modelo), ya que, la falta de información no permitirá reflejar el nivel de cumplimiento de los objetivos, dificultando en gran parte la toma oportuna de decisiones.

Finalmente, para fomentar los procesos de mejora, los directivos deben encaminar los esfuerzos hacia la alineación y participación conjunta de las áreas que conforman la organización. Contar con un sistema integrado que permita la generación de información oportuna y confiable, guiará a la institución hacia la consecución de los objetivos establecidos, cuyo propósito final se verá reflejado en la satisfacción de los pacientes, y de los miembros de la institución. La aplicación de este modelo en un hospital público, sirve como punto de partida, abriendo la posibilidad de ser aplicado en otros hospitales, y brinda información base de comparación para otras instituciones de salud.
Agradecimientos: Al personal médico y administrativo de los hospitales de Chile y Ecuador, entre ellos, al Director Académico del hospital, a la Directora zonal de salud, jefes departamentales de distintas unidades, personal de los departamentos de admisiones, consulta externa, epidemiología, emergencia, planificación y talento humano. Finalmente, agradecer al Proyecto Fondecyt Iniciación $\mathrm{N}^{\circ} 11160150$ como fuente de apoyo financiero de esta investigación.

\section{Referencias}

1. Valor J, Ribera J. (1990). Gestión en la empresa hospitalaria. Instituto de Estudios Superiores de la Empresa. IESE.

2. Artaza O, Barría MS, Fuenzalida A, Núñez K, Quintana A, Vargas I, Vidales A. (2005). Modelo de gestión de establecimientos hospitalarios. División de Gestión de Red Asistencial Subsecretaría de Redes Asistenciales. Chile: Ministerio de Salud, 90-100.

3. Organización Panamericana de la Salud. Indicadores de salud. Aspectos conceptuales y operativos. Washington, D.C.: OPS; 2018.

4. de Calidad Asistencial, S. E. (2011). Indicadores de calidad para hospitales del sistema nacional de salud. Oviedo, España: SECA.

5. Gabriel C, Da Costa M, Rossi F, Bernardes A, Miguelaci T, Prado M. Utilización de indicadores de desempeño en servicio de enfermería de hospital público. Rev Lat Am Enfermagem 2011; 19 (5): 1247-54.

6. Superintendencia de Salud. (2016). Guía Práctica para el Proceso de Acreditación de Prestadores Institucionales de Salud. Santiago. Recuperado de http://www.supersalud.gob.cl/observatorio/671/articles-8981_guia_practica_acreditacion.pdf.

7. De Estándares M. (2008). Indicadores e Instrumentos para medir la Calidad de la Atención Materno Neonatal del Ministerio de Salud Pública del Ecuador. MSP. Recuperado de https://cssr-ecuador.org/downloads/2016/07/5.-Manual-de-Estandares.pdf.

8. Instituto Nacional de Estadísticas y Censos (2018). Camas y Egresos Hospitalarios. Quito, Ecuador. Recuperado de https://www.ecuadorencifras.gob.ec/ camas-y-egresos-hospitalarios/

9. Armijos JC. (2017). Diseño e implementación de un modelo de indicadores de gestión para evaluar el desempeño de hospitales públicos.

10. Núñez A, Neriz L, Mateo R, Ramis F, Ramaprasad A. Emergency departments key performance indicators: A 
Indicadores de gestión para evaluar el desempeño de hospitales públicos - J. C. Armijos et al

unified framework and its practice. The International journal of health planning and management 2018; 33 (4): 915-33.

11. Nebot-Marzal CM, Mira-Solves JJ, Guilabert-Mora
M, Pérez-Jover V, Pablo-Comeche D, Quirós-Morató T, Peredo DC. Conjunto de indicadores de calidad y seguridad para hospitales de la Agencia Valenciana de Salud. Revista de Calidad Asistencial 2014; 29 (1): 29-35. 